\title{
G-Series Nerve Agent GD
}

National Cancer Institute

\section{Source}

National Cancer Institute. G-Series Nerve Agent GD. NCI Thesaurus. Code C161528.

A man-made highly toxic org anic phosphate nerve agent that is a clear, colorless liquid that gives off a colorless vapor and smells like camphor or rotting fruit, and is absorbed into the body by inhalation, ingestion, skin contact, or eye contact. GD inhibits acetylcholinesterase, increasing the neurotransmitter acetylcholine at central and peripheral neuronal synapses. 